\title{
Inclusion Properties of New Classes of Analytic Functions
}

\author{
Mohan Das, Ramesha Chendady, and Indira Kasarkod Pal \\ Department of Mathematics, Manipal Institute of Technology, Manipal, Karnataka 576 104, India
}

Correspondence should be addressed to Mohan Das; mohandas.ram@manipal.edu

Received 17 October 2013; Accepted 24 November 2013; Published 31 March 2014

Academic Editors: S. Deng and Y. Feng

Copyright (C) 2014 Mohan Das et al. This is an open access article distributed under the Creative Commons Attribution License, which permits unrestricted use, distribution, and reproduction in any medium, provided the original work is properly cited.

\begin{abstract}
The purpose of the present paper is to introduce certain new subclasses of analytic functions defined by Srivastava-Attiya operator and study their inclusion relationships and to obtain some interesting consequences of the inclusion relations.
\end{abstract}

\section{Introduction, Definitions, and Preliminaries}

Let $\mathbb{A}$ be the class of analytic functions $f(z)$ defined on the unit disk $E=\{z:|z|<1\}$, normalized by the conditions $f(0)=f^{\prime}(0)-1=0$. Let $S$ be the subclass of $\mathbb{A}$ consisting of univalent functions. Let $H$ denote the class of analytic convex univalent functions $h(z)$ defined in $E$ satisfying the conditions $h(0)=1$ and $\operatorname{Re}\{h(z)\}>0$ for all $z \in E$.

For any two analytic functions $f(z)$ and $g(z)$ defined in the unit disc $E$, we say that $f(z)$ is subordinate to $g(z)$ in $E$, denoted by $f(z) \prec g(z)$, if $g(z)$ is univalent in $E, f(0)=g(0)$ and $f(E) \subseteq g(E)$.

The convolution or the Hadamard product of two analytic functions with power series representations

$$
f(z)=\sum_{n=0}^{\infty} a_{n} z^{n}, \quad g(z)=\sum_{n=0}^{\infty} b_{n} z^{n}
$$

convergent in $|z|<1$ is the function $F(z)=f(z) * g(z)$, with power series representation

$$
F(z)=\sum_{n=0}^{\infty} a_{n} b_{n} z^{n}, \quad|z|<1
$$

The following classes of functions were defined by Shanmugam [1].
For a fixed analytic function $g(z) \in \mathbb{A}$ and $h(z) \in H$,

$$
\begin{gathered}
S_{g}(h)=\left\{f(z) \in \mathbb{A}: \frac{z(g * f)^{\prime}(z)}{(g * f)(z)}<h(z),\right. \\
\left.\frac{(g * f)(z)}{z} \neq 0\right\}, \\
C_{g}(h)=\left\{f \in \mathbb{A}: 1+\frac{z(g * f)^{\prime \prime}(z)}{(g * f)^{\prime}(z)}<h(z),\right. \\
\left.\frac{(g * f)(z)}{z} \neq 0\right\}, \\
K_{g}(h)=\left\{f(z) \in \mathbb{A}: \frac{z(g * f)^{\prime}(z)}{(g * \phi)(z)}<h(z),\right. \\
\left.\phi(z) \in S_{g}(h), \frac{(g * \phi)(z)}{z} \neq 0\right\} .
\end{gathered}
$$

We note that $f(z) \in C_{g}(h) \Leftrightarrow z f^{\prime}(z) \in S_{g}(h)$.

When $g(z)=z /(1-z)$ and $h(z)=(1+z) /(1-z)$, $S_{g}(h)=S^{*}$, the class of starlike functions; $C_{g}(h)=C$, the class of convex functions; and $K_{g}(h)=K$, the class of close to convex functions. 
The generalized Hurwitz-Lerch Zeta function $\phi(z, s, a)$ is defined as

$$
\phi(z, s, a)=\sum_{n=0}^{\infty} \frac{z^{n}}{(a+n)^{s}},
$$

where $a \in \mathbb{C} \backslash \mathbb{Z}_{0}^{-} ; s \in \mathbb{C}$, when $|z|<1$; Re $s>1$, when $|z|=$ 1 ; and $\mathbb{Z}_{0}^{-}=\mathbb{Z} \backslash \mathbb{N}$.

This function contains, as its special cases, functions such as the Riemann and Hurwitz zeta function, Lerch zeta function, the polylogarithmic function, and the Lipschitz Lerch zeta function.

Several interesting properties and characteristics of the Hurwitz-Lerch zeta function $\phi(z, s, a)$ can be found in the recent investigations by Choi and Srivastava [2], Ferreira and López [3], Garg et al. [4], Lin and Srivastava [5], and Lin et al. [6].

Using this function, Srivastava and Attiya [7] introduced the following family of linear operator $J_{\mu, b}: \mathbb{A} \rightarrow \mathbb{A}$, defined by

$$
\begin{gathered}
J_{\mu, b} f(z)=G_{\mu, b} * f(z), \\
\left(z \in E, b \in \mathbb{C} \backslash \mathbb{Z}_{0}^{-} ; \mu \in \mathbb{C}\right),
\end{gathered}
$$

where the function $G_{\mu, b}$ is given by

$$
\begin{aligned}
G_{\mu, b}(z) & =(1+b)^{\mu}\left[\phi(z, \mu, b)-b^{-\mu}\right] \\
& =z+\sum_{n=2}^{\infty}\left(\frac{b+1}{b+n}\right)^{\mu} z^{n}, \quad(z \in E) .
\end{aligned}
$$

Using (6) in (5), we get

$$
J_{\mu, b} f(z)=z+\sum_{n=2}^{\infty}\left(\frac{b+1}{b+n}\right)^{\mu} a_{n} z^{n}, \quad(z \in E) .
$$

For $f(z) \in \mathbb{A}$ and $z \in E$,

$$
\begin{gathered}
J_{0, b} f(z)=f(z), \\
J_{1,0} f(z)=\int_{0}^{z} \frac{f(t)}{t} d t=A(f), \\
J_{1, b} f(z)=\frac{1+b}{z^{b}} \int_{0}^{z} t^{b-1} f(t) d t=J_{b}(f),
\end{gathered}
$$

where $A(f)$ denotes Alexander transform [8] and $J_{b}(f)$ denotes Bernardi integral operator [9].

By (7), Srivastava and Attiya obtained the following relation:

$$
z\left(J_{\mu+1, b} f(z)\right)^{\prime}=(b+1) J_{\mu, b} f(z)-b J_{\mu+1, b} f(z) .
$$

In order to obtain our main results, we need the following lemmas.

Lemma 1 (see [10]). Let $\beta$ and $\gamma$ be constants. Let $\psi$ be convex univalent in $E$ with $\psi(0)=1$ and $\operatorname{Re}(\beta \psi(z)+\gamma)>0$ for $z$ in E. Let $p(z)$ be analytic in $E$ with $p(0)=1$. Then

$$
p(z)+\frac{z p^{\prime}(z)}{\beta p(z)+\gamma} \prec \psi(z) \text { implies } p(z) \prec \psi(z) .
$$

Lemma 2 (see [11]). Let $\beta$ and $\gamma$ be constants. Let $\psi$ be convex univalent in $E$ with $\psi(0)=1$ and $\operatorname{Re}(\beta \psi(z)+\gamma)>0$ for $z$ in $E$. Let $q(z)$ be analytic in $E$ with $q(z) \prec \psi(z)$. Let $p(z)$ be analytic in $E$ with $p(0)=1$. Then

$$
p(z)+\frac{z p^{\prime}(z)}{\beta q(z)+\gamma} \prec \psi(z) \text { implies } p(z) \prec \psi(z) .
$$

Lemma 3 (see [12]). Let a function $p(z)=1+p_{1} z+p_{2} z^{2}+\cdots$ be analytic in $E$ and $p(z) \neq 0, z \in E$. If there exists a point $z_{0} \in$ E such that $|\arg p(z)|<(\pi / 2) \alpha\left(|z|<\left|z_{0}\right|\right)$ and $\left|\arg p\left(z_{0}\right)\right|=$ $(\pi / 2) \alpha(0<\alpha \leq 1)$, then

$$
\frac{z_{0} p^{\prime}\left(z_{0}\right)}{p\left(z_{0}\right)}=i k \alpha
$$

where

$$
\begin{gathered}
k \geq \frac{1}{2}\left(a+\frac{1}{a}\right), \quad \text { when } \arg p\left(z_{0}\right)=\frac{\pi}{2} \alpha, \\
k \leq-\frac{1}{2}\left(a+\frac{1}{a}\right), \quad \text { when } \arg p\left(z_{0}\right)=-\frac{\pi}{2} \alpha,
\end{gathered}
$$

and $\left(p\left(z_{0}\right)\right)^{1 / \alpha}= \pm i a(a>0)$.

\section{Main Results}

First we will define a subclass $Q_{g}(\alpha, h)$ as follows.

Definition 4. For a fixed analytic function $g \in \mathbb{A}$ and a real $\alpha \geq 0$,

$$
\begin{aligned}
& Q_{g}(\alpha, h) \\
& =\left\{f \in \mathbb{A}: \alpha \frac{\left[z(g * f)^{\prime}(z)\right]^{\prime}}{(g * \psi)^{\prime}(z)}+(1-\alpha) \frac{z(g * f)^{\prime}(z)}{(g * \psi)(z)}\right. \\
& \left.\prec h(z), \psi(z) \in S_{g}(h)\right\} .
\end{aligned}
$$

Remark 5. When $g(z)=z /(1-z), \psi(z)=z$, and $h(z)=$ $(1+z) /(1-z), Q_{g}(\alpha, h)$ reduces to the class $R(\alpha)=\{f \in \mathbb{A}:$ $\left.\operatorname{Re}\left\{\alpha z f^{\prime \prime}(z)+f^{\prime}(z)\right\}>0\right\}$.

Theorem 6. For $\alpha>0, Q_{g}(\alpha, h) \subseteq K_{g}(h)$.

Proof. Let $f(z) \in Q_{g}(\alpha, h)$ and $p(z)=z(g * f)^{\prime}(z) /(g *$ $\psi)(z), \psi(z) \in S_{g}(h)$.

Note that $p(z)$ is analytic and $p(0)=0$.

Then,

$$
\begin{gathered}
p(z)(g * \psi)^{\prime}(z)+p^{\prime}(z)(g * \psi)(z)=\left[z(g * f)^{\prime}(z)\right]^{\prime} \\
\Longrightarrow \frac{\left[z(g * f)^{\prime}(z)\right]^{\prime}}{(g * \psi)^{\prime}(z)}=p(z)+p^{\prime}(z) \frac{(g * \psi)(z)}{(g * \psi)^{\prime}(z)} .
\end{gathered}
$$


Hence

$$
\begin{gathered}
\alpha \frac{\left[z(g * f)^{\prime}(z)\right]^{\prime}}{(g * \psi)^{\prime}(z)}+(1-\alpha) \frac{z(g * f)^{\prime}(z)}{(g * \psi)(z)} \prec h(z) \\
\Longrightarrow p(z)+\frac{\left(z p^{\prime}(z)\right)}{(1 / \alpha) q(z)} \prec h(z),
\end{gathered}
$$

where

$$
q(z)=\frac{\left(z(g * \psi)^{\prime}(z)\right)}{(g * \psi)(z)} .
$$

Since $\psi(z) \in S_{g}(h), q(z) \prec h(z)$.

Thus, by using Lemma 2 ,

$$
\frac{z(g * f)^{\prime}(z)}{(g * \psi)(z)}=p(z) \prec h(z) .
$$

That is, $f(z) \in K_{g}(h)$.

In the following, we obtain an inclusion relation of the class $Q_{g}(\alpha, h)$.

Theorem 7. For $0<\beta \leq \alpha, Q_{g}(\alpha, h) \subseteq Q_{g}(\beta, h)$.

Proof. Let $f(z) \in Q_{g}(\alpha, h)$.

Then

$$
\begin{array}{r}
\alpha \frac{\left[z(g * f)^{\prime}(z)\right]^{\prime}}{(g * \psi)^{\prime}(z)}+(1-\alpha) \frac{z(g * f)^{\prime}(z)}{(g * \psi)(z)} \in h(E), \\
z \in E, \psi \in S_{g}(h),
\end{array}
$$

and by Theorem 6 ,

$$
\frac{z(g * f)^{\prime}(z)}{(g * \psi)(z)} \prec h(z), \quad z \in E .
$$

Thus,

$$
\frac{z(g * f)^{\prime}(z)}{(g * \psi)(z)} \in h(E), \quad z \in E .
$$

Since $h(E)$ is a convex domain,

$$
\begin{aligned}
& t\left[\alpha \frac{\left[z(g * f)^{\prime}(z)\right]^{\prime}}{(g * \psi)^{\prime}(z)}+(1-\alpha) \frac{z(g * f)^{\prime}(z)}{(g * \psi)(z)}\right] \\
& +(1-t)\left[\frac{z(g * f)^{\prime}(z)}{(g * \psi)(z)}\right] \in h(E), \quad z \in E,
\end{aligned}
$$

where $0<t \leq 1$.

That is,

$$
\alpha t \frac{\left[z(g * f)^{\prime}(z)\right]^{\prime}}{(g * \psi)^{\prime}(z)}+(1-\alpha t) \frac{z(g * f)^{\prime}(z)}{(g * \psi)(z)} \in h(E),
$$

$$
z \in E \text {. }
$$

Let $\alpha t=\beta$. Then $0<\beta \leq \alpha$ and (23) implies that $f(z) \epsilon$ $Q_{g}(\beta, h)$.
Theorem 8. Let $n \in \mathbb{N}_{0}$. If $f \in S_{g}(h)$ then $J_{n, b}(f) \in S_{g}(h)$, for $\operatorname{Re} b \geq 0$.

Proof. Let $f(z) \in S_{g}(h)$.

We prove the result by mathematical induction on $n$.

When $n=0, J_{0, b}(f)=f \in S_{g}(h)$.

When $n=1$, set

$$
p(z)=z \frac{\left(J_{1, b}(f) * g\right)^{\prime}}{J_{1, b}(f) * g} .
$$

Clearly, $p(z)$ is analytic and $p(0)=1$.

From (9), we get

$$
\begin{gathered}
z\left(J_{1, b}(f) * g\right)^{\prime}=(b+1)(f * g)-b\left(J_{1, b}(f) * g\right) \\
\Longrightarrow p(z)+b=(b+1) \frac{f * g}{J_{1, b}(f) * g} .
\end{gathered}
$$

By logarithmic differentiation and multiplication by $z$, we get

$$
\frac{z p^{\prime}(z)}{p(z)+b}+p(z)=z \frac{(f * g)^{\prime}}{f * g} \prec h(z) .
$$

As Re $b \geq 0$, by Lemma 1 , we get $p(z) \prec h(z)$.

That is, $J_{1, b}(f) \in S_{g}(h)$.

Suppose that $J_{m, b}(f) \in S_{g}(h)$.

Set

$$
p(z)=z \frac{\left(J_{m+1, b}(f) * g\right)^{\prime}}{J_{m+1, b}(f) * g} .
$$

Clearly, $p(z)$ is analytic and $p(0)=1$.

From (9), we get

$$
\begin{aligned}
& z\left(J_{m+1, b}(f) * g\right)^{\prime} \\
& \quad=(b+1)\left(J_{m, b}(f) * g\right)-b\left(J_{m+1, b}(f) * g\right) \\
& \quad \Longrightarrow p(z)+b=(b+1) \frac{J_{m, b}(f) * g}{J_{m+1, b}(f) * g} .
\end{aligned}
$$

By logarithmic differentiation and multiplication by $z$, we get

$$
\frac{z p^{\prime}(z)}{p(z)+b}+p(z)=z \frac{\left(J_{m, b}(f) * g\right)^{\prime}}{J_{m, b}(f) * g} \prec h(z) \text {. }
$$

Hence, by Lemma $1, p(z) \prec h(z)$, for Re $b \geq 0$.

That is, $J_{m+1, b}(f) \in S_{g}(h)$, which proves the result.

Theorem 9. Let $n \in \mathbb{N}_{0}$. If $f \in C_{g}(h)$ then $J_{n, b}(f) \in C_{g}(h)$, for $\operatorname{Re} b \geq 0$.

Proof. Let $f(z) \in C_{g}(h)$.

Then

$$
\begin{aligned}
z f^{\prime}(z) \in S_{g}(h) & \Longrightarrow J_{n, b}\left(z f^{\prime}(z)\right)=z\left(J_{n, b}(f)\right)^{\prime}(z) \in S_{g}(h) \\
& \Longrightarrow J_{n, b}(f) \in C_{g}(h) .
\end{aligned}
$$


Remark 10. The Bernardi integral operator $L_{c}$ preserves both the classes $S_{g}(h)$ and $C_{g}(h)$ whenever Re $c \geq 0$.

Corollary 11. Let $n \in \mathbb{N}_{0}$, $\operatorname{Re} b \geq 0$, and $\operatorname{Re} c \geq 0$.

(i) If $f \in S_{g}(h)$ then $J_{n, b}\left(L_{c}\right)(f) \in S_{g}(h)$.

(ii) If $f \in C_{g}(h)$ then $J_{n, b}\left(L_{c}\right)(f) \in C_{g}(h)$.

Theorem 12. Let $f \in S_{g}(h)$ and

$$
\alpha=\min _{z \in E}\{\operatorname{Re} h(z)\} .
$$

Then

$$
\operatorname{Re}\left\{\frac{J_{n, b}(f) * g}{J_{n+1, b}(f) * g}\right\}>\frac{b+\alpha}{b+1} \quad \text { for } b>0 .
$$

Proof. Let $f \in S_{g}(h)$.

From (9), we get

$$
(b+1) \frac{J_{n, b}(f) * g}{J_{n+1, b}(f) * g}=b+z \frac{\left(J_{n+1, b}(f) * g\right)^{\prime}}{J_{n+1, b}(f) * g} .
$$

From Theorem $8, J_{n, b}(f) \in S_{g}(h)$, for all $n$.

Hence,

$$
(b+1) \frac{J_{n, b}(f) * g}{J_{n+1, b}(f) * g}-b=z \frac{\left(J_{n+1, b}(f) * g\right)^{\prime}}{J_{n+1, b}(f) * g} \prec h(z) .
$$

Let

$$
\alpha=\min _{z \in E}\{\operatorname{Re} h(z)\} .
$$

Then for $z \in E$,

$$
\operatorname{Re}\left\{(b+1) \frac{J_{n, b}(f) * g}{J_{n+1, b}(f) * g}-b\right\}>\alpha .
$$

This completes the proof.

In the following, we introduce certain classes of analytic functions defined by Srivastava-Attiya operator and investigate their inclusion relationships.

Let $h(z) \in H$ and $g(z) \in \mathbb{A}$.

Definition 13. For $\mu \in \mathbb{C}$ and $b \in \mathbb{C} \backslash \mathbb{Z}_{0}^{-}$, let

$$
\begin{aligned}
S_{g}(\mu, b ; h)= & \left\{f \in \mathbb{A}: J_{\mu, b} f(z) \in S_{g}(h)\right\} \\
& =\left\{f \in \mathbb{A}: \frac{z\left(J_{\mu, b}(f) * g\right)^{\prime}}{J_{\mu, b}(f) * g} \prec h(z)\right\} .
\end{aligned}
$$

Definition 14. For $\mu \in \mathbb{C}$ and $b \in \mathbb{C} \backslash \mathbb{Z}_{0}^{-}$, let

$$
\begin{aligned}
C_{g}(\mu, b ; h) & =\left\{f \in \mathbb{A}: J_{\mu, b} f(z) \in C_{g}(h)\right\} \\
& =\left\{f \in \mathbb{A}: 1+\frac{z\left(J_{\mu, b}(f) * g\right)^{\prime \prime}}{\left(J_{\mu, b}(f) * g\right)^{\prime}} \prec h(z)\right\} .
\end{aligned}
$$

Remark 15. Clearly, $f(z) \in C_{g}(\mu, b ; h)$ if and only if $z f^{\prime}(z) \in$ $S_{g}(\mu, b ; h)$.

Definition 16. For $\mu \in \mathbb{C}$ and $b \in \mathbb{C} \backslash \mathbb{Z}_{0}^{-}$, let

$K_{g}(\mu, b ; h)$

$$
=\left\{f \in \mathbb{A}: \frac{z\left(J_{\mu, b}(f) * g\right)^{\prime}}{J_{\mu, b}(\phi) * g} \prec h(z), \phi(z) \in S_{g}(\mu, b ; h)\right\} .
$$

Remark 17. We note that

$$
\begin{gathered}
S_{g}(0, b ; h)=S_{g}(h), \quad C_{g}(0, b ; h)=C_{g}(h), \\
K_{g}(0, b ; h)=K_{g}(h) .
\end{gathered}
$$

Theorem 18. If $\operatorname{Re} b \geq 0$, then $S_{g}(\mu, b ; h) \subset S_{g}(\mu+1, b ; h)$.

Proof. Let $f \in S_{g}(\mu, b ; h)$. Then,

$$
\frac{z\left(J_{\mu, b}(f) * g\right)^{\prime}}{J_{\mu, b}(f) * g} \prec h(z) .
$$

Let

$$
p(z)=\frac{z\left(J_{\mu+1, b}(f) * g\right)^{\prime}}{J_{\mu+1, b}(f) * g}
$$

where $p(z)$ is analytic and $p(0)=1$.

Using (42) in (9), we get

$$
b+p(z)=(b+1) \frac{\left(J_{\mu, b}(f) * g\right)}{J_{\mu+1, b}(f) * g} .
$$

On logarithmic differentiation and multiplication by $z$, we arrive at

$$
p(z)+\frac{z p^{\prime}(z)}{b+p(z)}=\frac{z\left(J_{\mu, b}(f) * g\right)^{\prime}}{J_{\mu, b}(f) * g} \prec h(z) .
$$

Since $h \in H$ is convex univalent in $E$ with $h(0)=1$ and $\operatorname{Re} h(z)>0$, Lemma 1 and the subordination relation (44) gives

$$
p(z) \prec h(z) \quad \text { for } \operatorname{Re} b \geq 0,
$$

proving the theorem.

Theorem 19. If $\operatorname{Re} b \geq 0$, then $C_{g}(\mu, b ; h) \subset C_{g}(\mu+1, b ; h)$.

Proof. Let $f \in C_{g}(\mu, b ; h)$.

Then

$$
\begin{gathered}
z f^{\prime}(z) \in S_{g}(\mu, b ; h) \subset S_{g}(\mu+1, b ; h) \\
\Longrightarrow f(z) \in C_{g}(\mu+1, b ; h)
\end{gathered}
$$

Theorem 20. If $\operatorname{Re} b \geq 0$, then $K_{g}(\mu, b ; h) \subset K_{g}(\mu+1, b ; h)$. 
Proof. Let $f(z) \in K_{g}(\mu, b ; h)$. Then, by Definition 16 , there exists a function $\phi(z) \in S_{g}(\mu, b ; h)$ such that

$$
\frac{z\left(J_{\mu, b}(f) * g\right)^{\prime}}{J_{\mu, b}(\phi) * g} \prec h(z) .
$$

Using Theorem 18, we get $\phi(z) \in S_{g}(\mu+1, b ; h)$.

Thus,

$$
q(z)=\frac{z\left(J_{\mu+1, b}(\phi) * g\right)^{\prime}}{J_{\mu+1, b}(\phi) * g(z)} \prec h(z)
$$

where $q(z)$ is analytic.

Let

$$
p(z)=\frac{z\left(J_{\mu+1, b}(f) * g\right)^{\prime}}{J_{\mu+1, b}(\phi) * g},
$$

where $p(z)$ is analytic and $p(0)=1$.

Now, making use of (9),

$$
\begin{aligned}
& \frac{z\left(J_{\mu, b}(f) * g\right)^{\prime}}{J_{\mu, b}(\phi) * g} \\
& =\frac{J_{\mu, b}\left(z f^{\prime}\right) * g}{J_{\mu, b}(\phi) * g} \\
& =\frac{z\left(J_{\mu+1, b}\left(z f^{\prime}\right) * g\right)^{\prime}+b J_{\mu+1, b}\left(z f^{\prime}\right) * g}{z\left(J_{\mu+1, b}(\phi) * g\right)^{\prime}+b J_{\mu+1, b}(\phi) * g} \\
& =\left(\frac{z\left(J_{\mu+1, b}\left(z f^{\prime}\right) * g\right)^{\prime}}{J_{\mu+1, b}(\phi) * g}+\frac{b J_{\mu+1, b}\left(z f^{\prime}\right) * g}{J_{\mu+1, b}(\phi) * g}\right) \\
& \quad \times\left(\frac{z\left(J_{\mu+1, b}(\phi) * g\right)^{\prime}}{J_{\mu+1, b}(\phi) * g}+b\right)^{-1} .
\end{aligned}
$$

Simplifying the above, using (48) and (49), and then using (47) we arrive at

$$
p(z)+\frac{z p^{\prime}(z)}{q(z)+b}=\frac{z\left(J_{\mu, b}(f) * g\right)^{\prime}}{J_{\mu, b}(\phi) * g} \prec h(z) .
$$

Since $h \in H$ is convex univalent in $E$ with $h(0)=1$ and $\operatorname{Re} h(z)>0$, Lemma 2 and the subordination relation (51) gives

$$
p(z) \prec h(z), \quad \text { for } \operatorname{Re} b \geq 0,
$$

proving the assertion.

Now, we consider certain interesting consequences of the above inclusion relationships.
Corollary 21. Suppose that $f \in \mathbb{A}$.

(i) If $A(f) \in S_{g}(h)$ then $f \in S_{g}(n, 0 ; h), n \in \mathbb{N} \backslash\{1\}$.

(ii) If $A(f) \in C_{g}(h)$ then $f \in C_{g}(n, 0 ; h), n \in \mathbb{N} \backslash\{1\}$.

(iii) If $A(f) \in K_{g}(h)$ then $f \in K_{g}(n, 0 ; h), n \in \mathbb{N} \backslash\{1\}$.

Corollary 22. Suppose that $f \in \mathbb{A}$ and $\operatorname{Re} c \geq 0$.

(i) If $L_{c}(f) \in S_{g}(h)$ then $f \in S_{g}(n, c ; h), n \in \mathbb{N} \backslash\{1\}$.

(ii) If $L_{c}(f) \in C_{g}(h)$ then $f \in C_{g}(n, c ; h), n \in \mathbb{N} \backslash\{1\}$.

(iii) If $L_{c}(f) \in K_{g}(h)$ then $f \in K_{g}(n, c ; h), n \in \mathbb{N} \backslash\{1\}$.

In a similar manner one can obtain many applications of Theorems 18, 19, and 20.

Using Srivastava-Attiya integral operator, we introduce some classes of analytic functions, analogous to the class of strongly starlike functions of order $\alpha$ and the class of strongly convex functions of order $\alpha$, and study the properties of the newly defined classes.

Let $0<\alpha \leq 1, n \in \mathbb{N}_{0}$, Re $b \geq 0, g \in \mathbb{A}$, and $z \in E$.

Definition 23. Let

$$
\begin{gathered}
\widetilde{S}_{n, b ; g}(\alpha) \\
=\left\{f \in \mathbb{A}:\left|\arg \left(\frac{z\left(J_{n, b}(f) * g\right)^{\prime}}{J_{n, b}(f) * g}\right)\right|<\frac{\pi}{2} \alpha,\right. \\
\left.\frac{z\left(J_{n, b}(f) * g\right)^{\prime}}{J_{n, b}(f) * g} \neq 0\right\} .
\end{gathered}
$$

Definition 24. Let

$$
\begin{aligned}
& \widetilde{C}_{n, b ; g}(\alpha) \\
& =\left\{f \in A:\left|\arg \left(1+\frac{z\left(J_{n, b}(f) * g\right)^{\prime \prime}}{\left(J_{n, b}(f) * g\right)^{\prime}}\right)\right|<\frac{\pi}{2} \alpha,\right. \\
& \left.1+\frac{z\left(J_{n, b}(f) * g\right)^{\prime \prime}}{\left(J_{n, b}(f) * g\right)^{\prime}} \neq 0\right\} .
\end{aligned}
$$

Remark 25. Clearly, $f \in \widetilde{C}_{n, b ; g}(\alpha)$ if and only if $z f^{\prime}(z) \in$ $\widetilde{S}_{n, b ; g}(\alpha)$.

Remark 26. For $n=0, g(z)=(1+z) /(1-z), \widetilde{S}_{0, b ; g}(\alpha)$ is the class of strongly starlike functions of order $\alpha$ and $\widetilde{C}_{0, b ; g}(\alpha)$ is the class of strongly convex functions of order $\alpha$.

Now we prove inclusion relationships for the above defined classes.

Theorem 27. Suppose that $b \geq 0$. Then $\widetilde{S}_{n, b ; g}(\alpha) \subset \widetilde{S}_{n+1, b ; g}(\alpha)$ for each $n, n \in \mathbb{N}_{0}$.

Proof. Let $f(z) \in \widetilde{S}_{n, b ; g}(\alpha)$.

We set

$$
\frac{\left(z J_{n+1, b}(f) * g\right)^{\prime}}{J_{n+1, b}(f) * g}=p(z)
$$


where $p(z)$ is analytic in $E, p(0)=1$ and $p(z) \neq 0$ for all $z \in E$.

Using (9) and (55), we have

$$
(b+1) \frac{J_{n, b}(f) * g}{J_{n+1, b}(f) * g}=b+p(z) .
$$

On logarithmic differentiation and multiplication by $z$, we get

$$
\begin{aligned}
\frac{z\left(J_{n, b}(f) * g\right)^{\prime}}{J_{n, b}(f) * g} & =\frac{z\left(J_{n+1, b}(f) * g\right)^{\prime}}{J_{n+1, g}(f) * g}+\frac{z p^{\prime}(z)}{b+p(z)} \\
& =p(z)+\frac{z p^{\prime}(z)}{b+p(z)} .
\end{aligned}
$$

Suppose that there exists a point $z_{0} \in E$ such that

$$
|\arg p(z)|<\frac{\pi}{2} \alpha \quad\left(|z|<\left|z_{0}\right|\right), \quad\left|\arg p\left(z_{0}\right)\right|=\frac{\pi}{2} \alpha .
$$

Then, applying Lemma 3, we can write

$$
\frac{z_{0} p^{\prime}\left(z_{0}\right)}{p\left(z_{0}\right)}=i k \alpha, \quad\left(p\left(z_{0}\right)\right)^{1 / \alpha}= \pm i a \quad(a>0) .
$$

Thus, if arg $p\left(z_{0}\right)=-(\pi / 2) \alpha$, then from (57)

$$
\begin{aligned}
& \frac{z_{0}\left(J_{n, b}\left(f\left(z_{0}\right)\right) * g\left(z_{0}\right)\right)^{\prime}}{J_{n, b}\left(f\left(z_{0}\right)\right) * g\left(z_{0}\right)} \\
& \quad=p\left(z_{0}\right)\left[1+\frac{z_{0} p^{\prime}\left(z_{0}\right) / p\left(z_{0}\right)}{b+p\left(z_{0}\right)}\right] \\
& =a^{\alpha} e^{-i \pi \alpha / 2}\left[1+\frac{i k \alpha}{b+a^{\alpha} e^{-i \pi \alpha / 2}}\right] .
\end{aligned}
$$

This implies that

$$
\begin{aligned}
& \arg \left\{\frac{z_{0}\left(J_{n, b}\left(f\left(z_{0}\right)\right) * g\left(z_{0}\right)\right)^{\prime}}{J_{n, b}\left(f\left(z_{0}\right)\right) * g\left(z_{0}\right)}\right\} \\
& =-\frac{\pi}{2} \alpha+\arg \left[1+\frac{i k \alpha}{b+a^{\alpha} e^{-i \pi \alpha / 2}}\right] \\
& =-\frac{\pi}{2} \alpha \\
& \quad+\tan ^{-1}\left[\frac{k \alpha\left(b+a^{\alpha} \cos (\pi \alpha / 2)\right)}{a^{2 \alpha}+b^{2}+a^{\alpha}(2 b \cos (\pi \alpha / 2)-k \alpha \sin (\pi \alpha / 2))}\right] \\
& \leq-\frac{\pi}{2} \alpha \quad\left(\text { where } k \leq-\frac{1}{2}\left(a+\frac{1}{a}\right) \leq-1\right),
\end{aligned}
$$

which contradicts the condition that $f(z) \in \widetilde{S}_{n, b ; g}(\alpha)$.

Similarly, if $\arg p\left(z_{0}\right)=(\pi / 2) \alpha$, then we obtain that

$$
\arg \left\{\frac{z_{0}\left(J_{n, b}\left(f\left(z_{0}\right)\right) * g\left(z_{0}\right)\right)^{\prime}}{J_{n, b}\left(f\left(z_{0}\right)\right) * g\left(z_{0}\right)}\right\} \geq \frac{\pi}{2} \alpha,
$$

which also contradicts the condition that $f(z) \in \widetilde{S}_{n, b ; g}(\alpha)$.
Thus we have

$$
|\arg p(z)|<\frac{\pi}{2} \alpha, \quad z \in E .
$$

That is,

$$
\begin{gathered}
\left|\arg \left\{\frac{z\left(J_{n+1, b}(f) * g\right)^{\prime}}{J_{n+1, g}(f) * g}\right\}\right|<\frac{\pi}{2} \alpha, \quad z \in E . \\
\Longrightarrow f(z) \in \widetilde{S}_{n+1, b ; g}(\alpha) .
\end{gathered}
$$

Theorem 28. Suppose that $b \geq 0$. Then $\widetilde{C}_{n, b ; g}(\alpha) \subset$ $\widetilde{C}_{n+1, b ; g}(\alpha)$, for $n \in \mathbb{N}_{0}$.

Proof. Let $f(z) \in \widetilde{C}_{n, b ; g}(\alpha)$.

Then

$$
\begin{aligned}
& z f^{\prime}(z) \in \widetilde{S}_{n, b ; g}(\alpha) \\
\Longrightarrow & z f^{\prime}(z) \in \widetilde{S}_{n+1, b ; g}(\alpha) \\
\Longrightarrow & f(z) \in \widetilde{C}_{n+1, b ; g}(\alpha) .
\end{aligned}
$$

\section{Conflict of Interests}

The authors declare that there is no conflict of interests regarding the publication of this paper.

\section{References}

[1] T. N. Shanmugam, "Convolution and differential subordination," International Journal of Mathematics and Mathematical Sciences, vol. 12, pp. 333-340, 1989.

[2] J. Choi and H. M. Srivastava, "Certain families of series associated with the Hurwitz-Lerch Zeta function," Applied Mathematics and Computation, vol. 170, no. 1, pp. 399-409, 2005.

[3] C. Ferreira and J. L. López, "Asymptotic expansions of the Hurwitz-Lerch zeta function," Journal of Mathematical Analysis and Applications, vol. 298, no. 1, pp. 210-224, 2004.

[4] M. Garg, K. Jain, and H. M. Srivastava, "Some relationships between the generalized Apostol-Bernoulli polynomials and Hurwitz-Lerch Zeta functions," Integral Transforms and Special Functions, vol. 17, no. 11, pp. 803-815, 2006.

[5] S.-D. Lin and H. M. Srivastava, "Some families of the HurwitzLerch Zeta functions and associated fractional derivative and other integral representations," Applied Mathematics and Computation, vol. 154, no. 3, pp. 725-733, 2004.

[6] S.-D. Lin, H. M. Srivastava, and P.-Y. Wang, "Some expansion formulas for a class of generalized Hurwitz-Lerch Zeta functions," Integral Transforms and Special Functions, vol. 17, no. 11, pp. 817-827, 2006.

[7] H. M. Srivastava and A. A. Attiya, "An integral operator associated with the Hurwitz-Lerch Zeta function and differential subordination," Integral Transforms and Special Functions, vol. 18 , no. 3, pp. 207-216, 2007. 
[8] J. W. Alexander, "Functions which map the interior of the unit circle upon simple regions," Annals of Mathematics, vol. 17, pp. 12-22, 1915-1916.

[9] S. D. Bernardi, "Convex and starlike univalent functions," Transactions of the American Mathematical Society, vol. 135, pp. 429-446, 1969.

[10] P. Eeningenburg, S. S. Miller, P. T. Mocanu, and M. O. Reade, "On a briot boquet differential subordination," in General Inequalities 3: 3rd International Conference on General Inequalities, vol. 64, pp. 339-348, 1983.

[11] K. S. Padmanabhan and R. Parvatam, "Some applications of differential subordination," Bulletin of the Australian Mathematical Society, vol. 37, pp. 321-330, 1985.

[12] M. Nunokawa, "On the order of strongly starlikeness of strongly convex functions," Proceedings of the Japan Academy A, vol. 69, pp. 234-237, 1993. 


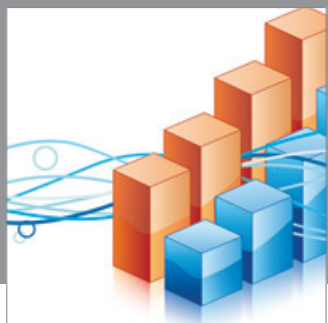

Advances in

Operations Research

mansans

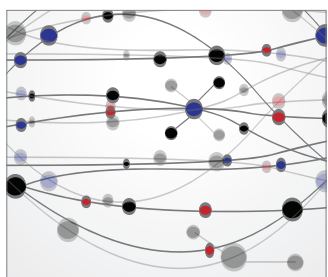

The Scientific World Journal
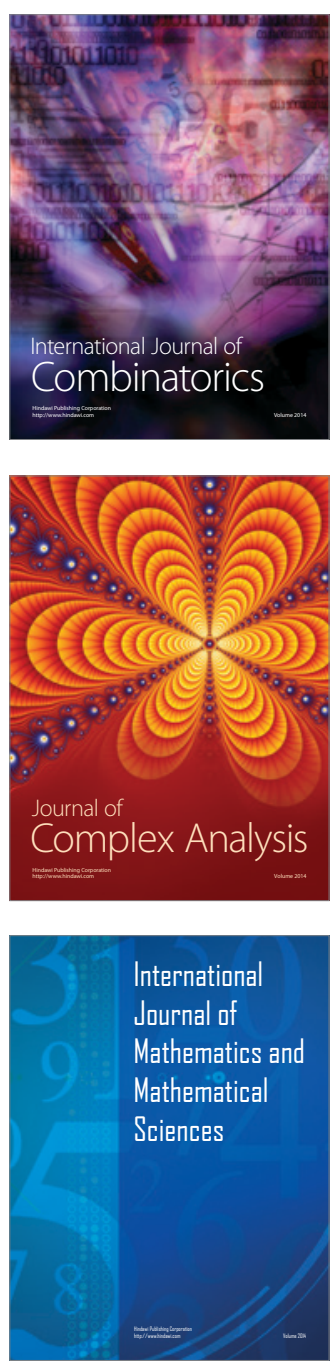
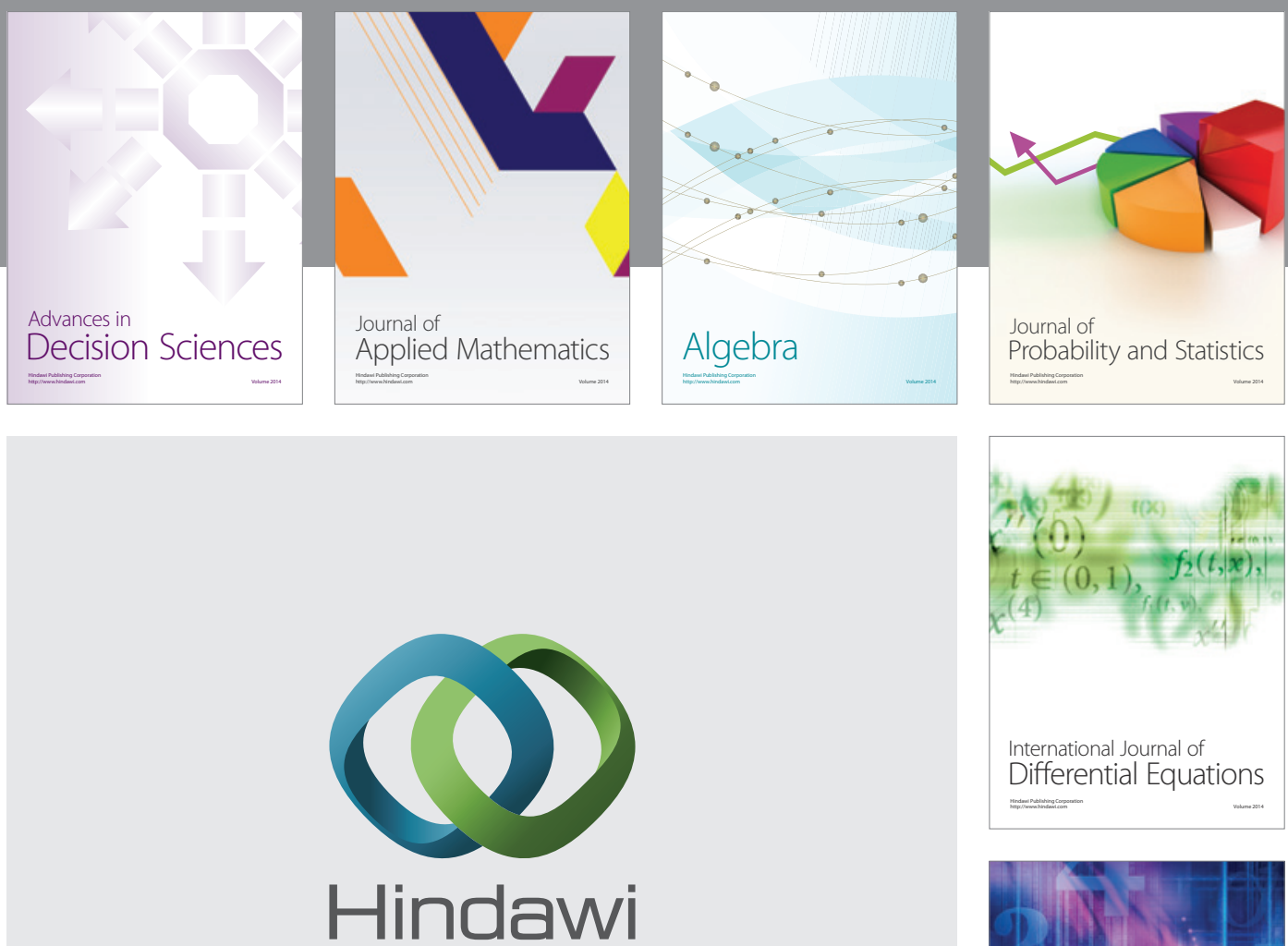

Submit your manuscripts at http://www.hindawi.com
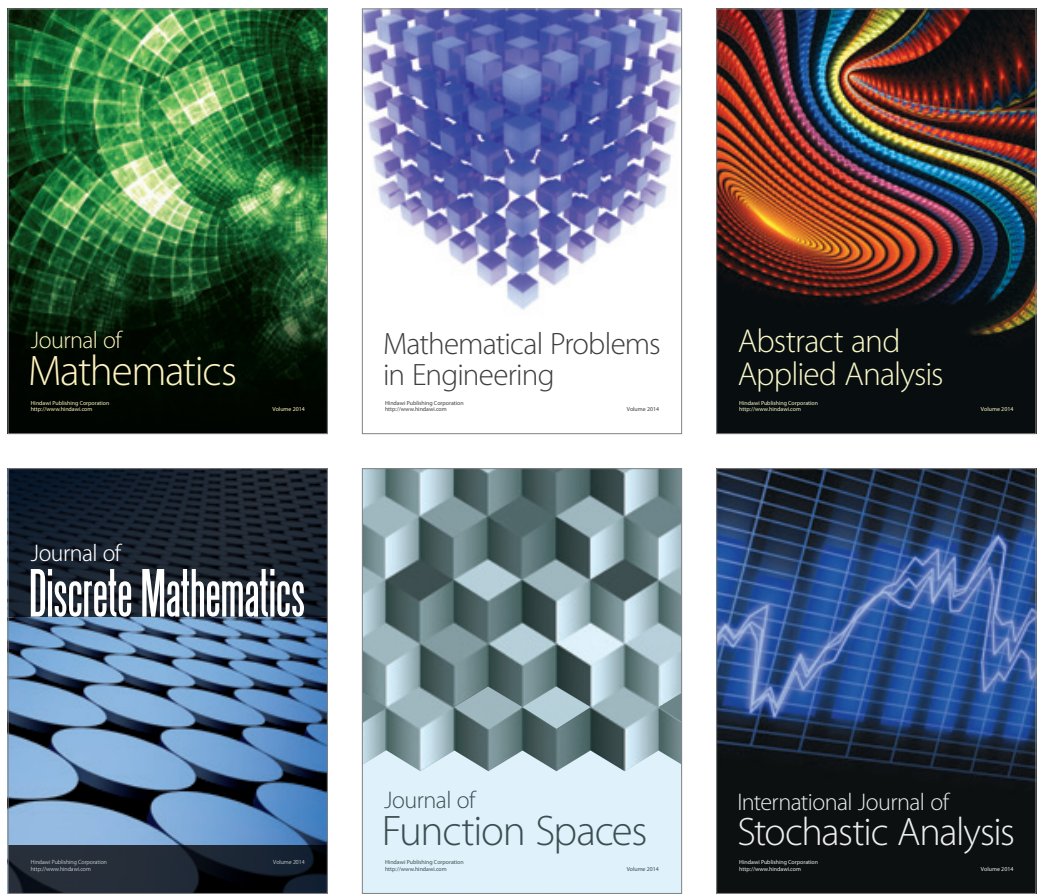

Journal of

Function Spaces

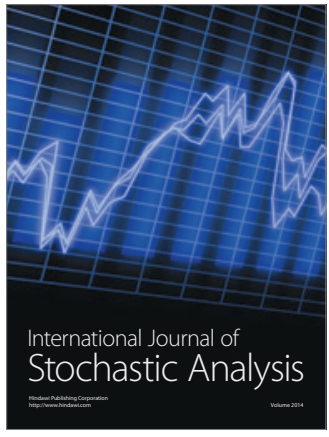

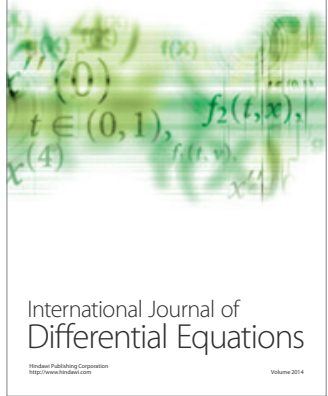
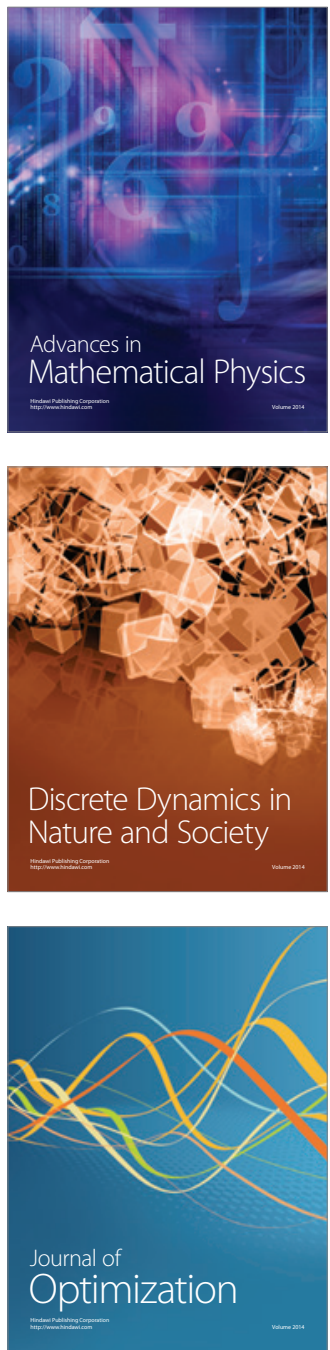